\title{
Improving the quality of care for inflammatory bowel disease
}

\author{
Byong Duk Ye ${ }^{1}$, Simon Travis ${ }^{2}$ \\ ${ }^{I}$ Department of Gastroenterology and Inflammatory Bowel Disease Center, Asan Medical Center, University of Ulsan College of Medicine, Seoul, \\ Korea; ${ }^{2}$ Translational Gastroenterology Unit, Oxford University Hospitals, Oxford, UK
}

Great strides have been achieved in the development of quality-of-care measures and standards for inflammatory bowel disease (IBD) over the last decade. The central structural component of care in IBD revolves around the multidisciplinary team, which should be equipped with the necessary resources to operate and implement decisions. Process measures have been defined by interest groups and can be adapted into process tools for the delivery of care for various patient subgroups and clinical scenarios. The emerging treat-to-target approach to IBD management may be used to achieve optimal long-term and holistic patient-centred outcomes, such as survival, control of inflammation and disease progression, symptomatic remission, quality of life and complications. Other important quality-of-care outcome measures for IBD include disutility of care, healthcare utilization and other patient-reported outcomes such as nutritional status and impact of fistulae. The current challenge for healthcare providers and health systems is the integration of quality-of-care structures and processes into clinical practice, and the consistent delivery of updated evidence-based quality IBD care to various patient populations by individual health care providers. Finally, the awareness and appreciation for quality of care in IBD is increasing in Asia, and Asian healthcare institutions should be encouraged to take the lead in improving the quality of care in IBD. (Intest Res 2019;17:45-53)

Key Words: Inflammatory bowel disease; Quality of care; Health system; Treat to target

\section{INTRODUCTION}

Improving the quality of care in any healthcare system is not a project, initiative, or even a goal, but is instead a continuous process that should be integral to the organization and healthcare delivery. That said, the spotlight has fallen on the quality of care in IBD due to the wide variation in practice indicating inconsistency in care (which extends beyond selecting the most appropriate drug) and the presence of a wide therapeutic gap in the efficacy of current treatments. ${ }^{1,2}$

To improve care, quality must be measured. The framework

Received August 5, 2018. Revised October 5, 2018. Accepted October 5, 2018. Correspondence to Byong Duk Ye, Department of Gastroenterology and Inflammatory Bowel Disease Center, Asan Medical Center, University of Ulsan College of Medicine, 88 Olympic-ro 43-gil, Songpa-gu, Seoul 05505, Korea. Tel: +82-2-3010-3181, Fax: +82-2-476-0824,

E-mail: bdye@amc.seoul.kr

ORCID Byong Duk Ye (https://orcid.org/0000-0001-6647-6325) by which this is achieved defines 3 components: structure, process and outcome measures. ${ }^{3}$ Until 2017, there was no common language of healthcare outcome measures for IBD that had been agreed between patients, their associations and multidisciplinary specialists. ${ }^{4}$ However, the language of outcomes for IBD has now been agreed as part of the International Consortium for Health Outcomes Measurement (ICHOM, www.ichom.org), an international initiative covering $50 \mathrm{com}-$ mon disease areas. Hence, it is timely to review approaches to improve the quality of care for IBD. Action for improving the quality of IBD care should not be delayed any more, especially in Asia, where it is expected to have the largest burden of IBD in the near future.

\section{WHY IS QUALITY OF CARE IMPORTANT IN IBD?}

One of the commonly quoted definitions of quality in health- 
care is the one by the Institute of Medicine, defining it as "the degree to which health care services for individuals and populations increase the likelihood of desired outcomes and are consistent with current professional knowledge. ${ }^{n 5}$ IBD including CD and UC are chronic immune-mediated intestinal diseases characterized by chronic inflammation leading to progressive bowel damage and a significant burden of disease for patients and their families, often with poor long-term outcomes in terms of morbidity, surgery, hospitalization and quality of life. ${ }^{6}$ Quality of care in IBD is important because its improvement should lead to improvement of all the aforementioned clinical and quality-of-care outcomes.

There is much room for improvement in the quality of care in IBD. A study in Boston, Massachusetts, USA found that $77 \%$ of patients seeking a second opinion at a specialist center had received steroids for more than 3 months and of those treated with aminosalicylates, $66 \%$ were on suboptimal doses while $75 \%$ with distal colitis had not received topical therapy. ${ }^{7}$ Improving quality of care could also reduce such inconsistencies and variability in care and outcomes among regions and centres.

Additionally, the high cost of IBD to payors and society underscores the need for quality of care in this area. The total economic burden of UC, including welfare and indirect costs to society, has been estimated at $\$ 8.1$ to $\$ 14.9$ billion annually in the United States and $€ 12.5$ to $€ 29.1$ billion in Europe. ${ }^{8}$ Of these total direct costs (including consultations, drugs, hospitalisation and surgery) amount to $\$ 3.4$ to $\$ 8.6$ billion in the United States and $€ 5.4$ to $€ 12.6$ billion in Europe, with direct costs increasing with worsening disease severity. Improvement in quality of care could potentially reduce the economic burden of IBD.

\section{WHAT IS THE PROCESS OF IMPROVING QUALITY OF CARE?}

In order to improve quality of care in IBD, it is useful to set specific objectives and measurable goals to facilitate change and action. This can be done using the conceptual framework proposed by Donabedian, where quality of health care can be analyzed from 3 components: structure, process and outcomes (Fig. 1). ${ }^{3}$ Structure is the construct within which care is delivered, including hospital buildings, staff, finance and equipment. Process includes the transactions between patients and providers during the delivery of healthcare. Finally, outcomes refer to the effects of healthcare on the health status of patients and populations. This cascade of factors and the information

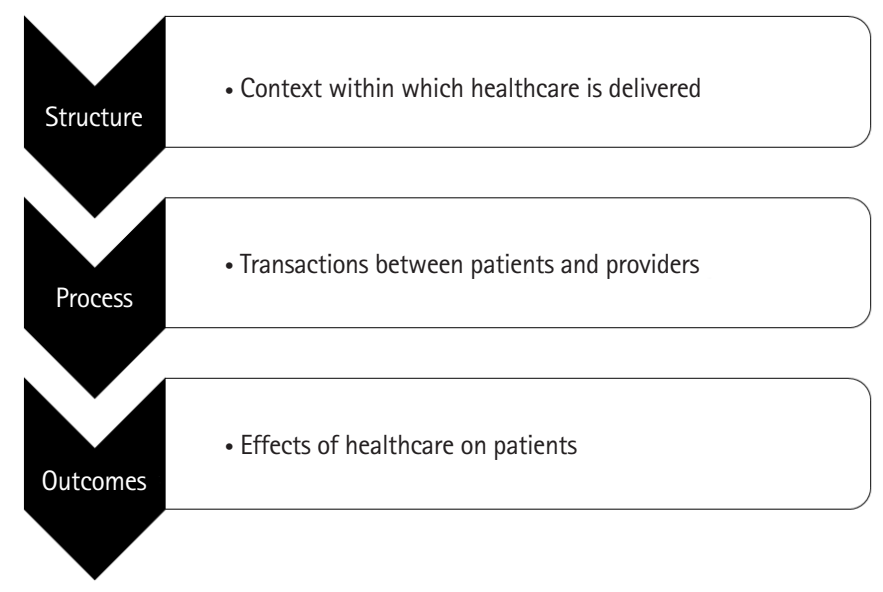

Fig. 1. The Donabedian framework for evaluating quality of healthcare.

about the detailed interactions between them are what enables analysis and improved quality of care to be delivered.

Structure of care delivery is important for chronic diseases, particularly for IBD, as caring for patients with IBD is resourceintensive, requiring access to both inpatient and outpatient facilities, endoscopy, imaging, laboratories and multidisciplinary teams (MDT). The allocation of resources to meet these structural needs is a process involving patient and healthcare practioner advocacy, resource planning and investment. For the purpose of this review, we will focus on the processes and outcomes where we think improvements can be delivered within short time frames and without large investments.

Process meaures are important in improving quality of care because they can be monitored frequently and changes in process measures can be correlated with both positive and negative outcomes, providing insight into what works and what does not work, which may lead to changes in structure.

Outcome measures are central to delivering value-based care, where value is the product of the outcome (defined by the patient) divided by the cost of delivering that care. ${ }^{9}$

\section{HOW CAN PATIENTS HELP?}

The involvement of patients in improving care in IBD can be in several areas:

- Shared decision-making in treatment decisions: This includes discussion about the choice of treatments (e.g., thiopurines, biologics or surgery) and allows more information to emerge for better decisions. There is some evidence that shared decision-making is associated with greater satisfac- 
tion and adherence in the use of biologics in rheumatoid arthritis, whereas in IBD, shared decision-making has been associated with greater satifaction and lower anxiety. ${ }^{10,11}$

- Improved communication between patients and healthcare practitioners: The patient is central to improving patient satisfaction, and communication can be facilitated by an IBD specialist nurse, educational apps or websites that feature frequently asked questions. Currently, more than 50 mobile applications related to IBD are available from 2 marketplaces, Google Play for Android and Apple's iTunes App store. ${ }^{12}$ These apps have potential benefits in improving quality of care in terms of patient education, remote disease monitoring, earlier intervention, improved adherence, patient empowerment, among others.

- Feedback from patients in improving services in IBD care: While health outcomes may often be slow to respond to structural changes and outcome measurement can be costly and time-consuming, engaging patients in the collection of their health data may facilitate feedback on the impact of quality improvements. ${ }^{13}$ Feedback helps drive improvement in quality, which is why the private sector (e.g., retailers, hoteliers) demand it often. Feedback can be acquired through feedback forms, surveys in IBD clinics, patient interviews, or more formalised involvement in planning structure and process improvements.

\section{WHAT ARE THE KEY STRUCTURAL MEASURES FOR QUALITY OF CARE?}

The central structural component of care in IBD is the MDT, owing to the complexity of $\mathrm{CD}$ and UC, which are associated with extra-intestinal manifestations or complications needing specialist surgeons, radiologists, pathologists and other healthcare professionals. ${ }^{14}$ The members of the MDT varies according to the complexity of the care being delivered and individual patient needs. However, for efficient healthcare delivery, it should include at least an IBD specialist-gastroenterologist, a surgeon, a radiologist, a pathologist, an IBD specialist nurse, a dietitian and a pharmacist-with defined links to named specialists in psychology, dermatology, rheumatology and ophthalmology. ${ }^{15,16}$

To be functional, the IBD MDT should have a dedicated space for meetings, with administrative support to document and implement decisions. Other structural components in-

Table 1. Key Components of Structure and Process for an IBD MDT

\begin{tabular}{|c|c|}
\hline \multirow[t]{2}{*}{ MDT } & Regular meetings, documented \\
\hline & Regional network of other IBD centres \\
\hline \multirow{2}{*}{ Patient engagement } & Support group \\
\hline & Involvement in the planning and (re)design of services \\
\hline \multirow[t]{6}{*}{ Outpatients } & Follow-up options (clinic visits, telephone, shared care or virtual clinic) \\
\hline & Vaccination program \\
\hline & Biological or immunomodulator monitoring program \\
\hline & Surveillance program \\
\hline & Sufficient toilet facilities \\
\hline & Education program \\
\hline \multirow[t]{5}{*}{ Inpatients } & Automatic contact or transfer of care protocol agreed with ED \\
\hline & Drug protocols shared with ED \\
\hline & Specialist or designated ward for patients with IBD, including sufficient toilets \\
\hline & Joint management with surgeons for acute severe colitis \\
\hline & Venous thromboembolism assessment and prophylaxis \\
\hline \multirow[t]{4}{*}{ Care pathways } & For diagnosis \\
\hline & For treatment of active UC or CD and monitoring \\
\hline & For treatment of UC or CD in remission and monitoring \\
\hline & For acute severe colitis \\
\hline
\end{tabular}

MDT, multidisciplinary team; ED, emergency department.

Adapted from Calvet $X_{1}$ et al. J Crohns Colitis 2014;8:240-251. ${ }^{17}$ 
clude adequate facilities for the needs of patients with IBD, such as sufficient toilets, designated IBD clinics, colorectal surgery clinics at the same time and next to IBD clinics, as well as nearby access to endoscopy, imaging and a specialist radiologist for advice (Table 1$).^{17}$

\section{WHAT ARE THE KEY PROCESS MEASURES NEEDED TO IMPROVE QUALITY OF CARE?}

Process measures should be evidence based (or failing that, should be guideline or consensus based), relatively easy to track and measure, and linked to desired outcomes. The Crohn's and Colitis Foundation of America (CCFA) have defined some quality process indicators for the management of patients with IBD, as shown in Table $2 .{ }^{6}$ However, some modifications are appropriate for Asia. For example, thiopurine S-methyltransferase (TPMT) genetic testing is not useful before starting azathiopurine in Asian patients, but testing for NUTD15 genotype should be done as it predicts the risk of leukopenia. ${ }^{18,19}$
Achievement of some process indicators is poor in the United States. A cross-sectional audit of the Optum Clinformatics Data Mart database, a large US claims database, reported that performance ranged from $0.4 \%$ for prophylaxis for venous thromboembolism (VTE), 67\% for testing for Clostridium difficile during a relapse, $51 \%$ for appropriate corticosteroid-sparing therapy, and $45 \%$ testing for latent tuberculosis (TB) before initiating anti-TNF therapy. ${ }^{20}$ Independent audit data is not available in Asia, but a survery on self-percieved attainment of the CCFA measures among 353 Asian physicans attending the Asian Organization for Crohn's and Colitis Congress in 2014 reported that the performace of documentation of IBD and screening for TB or hepatitis B before anti-TNF therapy was over $80 \%{ }^{21}$ However, other measures such as use of VTE prophylaxis in inpatients or vaccination against Pneumococcus sp. were performed in only around $20 \%$. It should also be noted that these are self-reported data, which has poor reliability as physicians almost always overestimate their delivery of healthcare. $^{22}$

Table 2. The Crohn's and Colitis Foundation of America's Top 10 Quality Process Indicators for IBD

\footnotetext{
Treatment

IF a patient with IBD is initiating anti-TNF therapy, THEN tuberculosis risk assessment should be documented, and tuberculin skin testing or interferon gamma release assay should be performed

IF a patient with IBD is initiating therapy with anti-TNF, THEN risk assessment for HBV should be documented

IF a patient with IBD requires at least $10 \mathrm{mg}$ prednisone (or equivalent) for 16 weeks or longer, THEN an appropriately dosed steroid-sparing agent ${ }^{\mathrm{a}}$ or operation should be recommended

IF a hospitalized patient with severe colitis is not improving on intravenous steroids within 3 days, THEN sigmoidoscopy with biopsy should be performed to exclude cytomegalovirus, AND surgical consultation should be obtained

IF a patient in whom a flare of IBD is suspected with new or worsening diarrhea THEN the patient should undergo Clostridium difficile testing at least once

IF a patient with IBD is initiating 6 MP/AZA, THEN TPMT testing should be performed before starting therapy

Surveillance

IF a patient with UC is found to have confirmed low-grade dysplasia in flat mucosa, THEN proctocolectomy or repeat surveillance within 6 months should be offered

IF a patient with extensive ${ }^{b}$ UC or CD involving the colon has had their disease for 8 to 10 years, THEN surveillance colonoscopy should be performed every 1 to 3 years

Health care maintenance

IF a patient with IBD is on immunosuppressive therapy, THEN patients should be educated about appropriate vaccinations, including (1) annual inactivated influenza, (2) pneumococcal vaccination with a 5-year booster, and (3) general avoidance of live virus vaccines

IF a patient with CD is an active tobacco smoker, THEN smoking cessation should be recommended, and treatment should be offered or suitable referral provided at least annually

${ }^{a} 6$-Mercaptopurine, 1.0 to $1.5 \mathrm{mg} / \mathrm{kg}$ daily; azathioprine, 2.0 to $2.5 \mathrm{mg} / \mathrm{kg}$ daily (if normal TPMT metabolism); methotrexate $25 \mathrm{mg}$ injected subcutaneously weekly, or appropriately dosed biological therapy.

'Left-sided for UC, or $1 / 3$ or more for CD.

'IF a patient with UC has co-existing primary sclerosing cholangitis (of any duration), THEN surveillance colonoscopy should be performed every 1 to 3 years. 6 MP, 6-mercaptopurine; AZA, azathioprine; TPMT, thiopurine methyltransferase.

Adapted from Melmed GY, et al. Inflamm Bowel Dis 2013;19:662-668, with permission from Oxford University Press. ${ }^{6}$
} 


\section{WHAT IS THE ROLE OF THE PROCESS MEA- SURES OF CLINICAL PATHWAYS AND CHECK- LISTS IN ENSURING CONSISTENCY IN CARE?}

One of the challenges associated with managing $\mathrm{CD}$ and UC is the consistent delivery of care that reflects the latest research to various patient populations by individual health care providers. Local clinical care pathways based on carefully constructed guidelines relevant to the local population following an approved process help provide guidance, ${ }^{23,24}$ but too often the guidelines are not followed. Interactive guidelines relating to clinical dilemmas, such as the European Crohn's and Colitis Organisation e-guide (http://www.e-guide.ecco-ibd.eu) may improve uptake of guidelines in practice.

In addition to promoting consistency in care, clinical pathways can also improve care by reducing the time to diagnosis and referral from the general practitioner to the specialist gastroenterologist, promoting tight monitoring to therapeutic targets, improving consistency in initaiting steroid-sparing therapies and outlining perioperative management. While studies that supports the introduction of guidelines in IBD are few, a retrospective review of admissions for IBD in St. Vincent's Hospital in Dublin showed that the introduction of a structured care pathway was associated with a reduction in hospital stay and an increase in VTE prophylaxis rates. ${ }^{25}$

Checklists are another tool for improving consistency and performance meaures in IBD care. They have a role in improving vaccination rates and $\mathrm{TB}$ screening prior to biologic initiation. ${ }^{26}$

\section{HOW CAN WE IMPROVE CLINICAL OUTCOMES THROUGH THE PROCESS MEASURES OF TIGHT MONITORING AND DISEASE CONTROL (TREAT- TO-TARGET)?}

The paradigm for treating IBD is changing focus from achieving clinical response and remission to more objective and predictive targets, such as corticosteroid-free remission and mucosal healing. The trend in IBD is towards a treat-to-target strategy similar to that in rheumatoid arthritis. ${ }^{27}$ This reflects a realisation that the ultimate goal of IBD management should be not just symptomatic remission but a more holistic goal that includes control of inflammation, optimising quality of life, maintaining symptom remission, stopping disease progression, and preventing progressive bowel damage and attendant complications.

This paradigm shift is facilitated by the availability of more powerful biologic agents that enable physicians to achieve deeper levels of inflammation control. Studies have shown that patients with mucosal healing were more likely to remain in remission and have a lower risk of hospitalization or complications needing surgery. For example, in the ACCENT I trial of infliximab for moderate to severe $\mathrm{CD}$, mucosal healing at weeks 10 and 54 was associated with a trend towards fewer hospitalizations and surgery. ${ }^{28}$ Similarly, the Norwegian IBSEN cohort study on UC showed that patients with mucosal healing 1 year after diagnosis had significantly lower colectomy rates at follow-up $(P=0.02){ }^{29}$ Furthermore, mucosal healing is a good therapeutic target because it is potentially objective (depending on its definition), it correlates with underlying bowel inflammation, and is predictive of long-term outcomes. ${ }^{30}$

The Selecting Therapeutic Targets in Inflammatory Bowel Disease (STRIDE) consensus group of the International Organization for the Study of Inflammatory Bowel Diseases has listed the therapeutic target for $\mathrm{CD}$ as the following: ${ }^{31}$

- Clinical and patient-reported remission, defined as resolution of abdominal pain and diarrhoea or altered bowel habit (assessed at least every 3 months until resolution, then every 6 to 12 months), plus;

- Endoscopic remission, defined as resolution of ulceration at ileocolonoscopy, or resolution of inflammation on crosssectional imaging, in patients who could not be assessed adequately by ileocolonoscopy (assessed at least every 6 to 9 months during active disease).

Similarly for UC the targets are as follows: ${ }^{31}$

- Clinical and patient-reported remission, defined as resolution of rectal bleeding and diarrhoea or altered bowel habit (assessed at least every 3 months until resolution, then every 6 to 12 months), plus;

- Endoscopic remission, defined as a Mayo endoscopic subscore or UC Endoscopic Index of Severity score of 0 or 1 (assessed 3 to 6 months after starting therapy in symptomatic patients and then at least every 3 months during active disease).

The treat-to-target paradigm was proposed as a way to achieve deep and sustained control of intestinal inflammation to reduce the risk of bowel damage and complications. By setting a target for monitoring and adjusting treatment to achieve that target, it was hoped that there would be better control of inflammation. The outstanding question is whether the benefits of treatment decisions based on targets alone (such as escalating treatment in an asymptomatic patient with endoscopic 
disease activity) exceed the risks, although initial evidence suggest that it does. In the CALM study, 244 patients with a $\mathrm{CD}$ and a mean disease duration of just 1 year were randomised to either 1 of 2 algorithms: (1) tight control based on activity indices, prednisone use, CRP and fecal calprotectin; or (2) clinical management based on activity indices and prednisone use. ${ }^{32}$ Monitoring therapy using both clinical parameters and biomarkers of inflammation led to superior endoscopic and clinical outcomes after 48 weeks compared with clinical parameter-driven decisions alone, without an increase in adverse events in the tight control group.

Other studies that have deployed a treat-to-target approach also validate this approach to treating IBD. In the REACT-1 study, patients randomized to early combined immunosuppression with adalimumab and azathioprine and regular 12weekly monitoring with a target of clinical remission defined as a Harvey-Bradshaw Index of 4 or lower had a lower risk of an adverse clinical outcome, i.e., surgery, hospital admission or serious disease-related complication (HR, 0.73 ; 95\% CI, $0.62-$ $0.86 ; P=0.003) .{ }^{33}$ Additionally, a retrospective study evaluated treat to target in a gastroenterology center from 2011 to 2012, where patients with CD patients had tight monitoring with planned endoscopies and therapeutic adjustments over a median follow-up period of 62 weeks. ${ }^{34}$ The study reported that 34 patients $(51 \%)$ had mucosal healing and 41 patients $(61 \%)$ had endoscopic improvement, and that the cumulative probabilities of mucosal healing were $13 \%$ and $45 \%$ at 24 and 52 weeks of treatment, respectively. Factors associated with mucosal healing included a interval between endoscopic procedures of less than 26 weeks (HR, 2.35; 95\% CI, 1.15-4.97; $P=0.035$ ) and adjustments to medical therapy when mucosal healing was not observed (HR, 4.28; 95\% CI, 1.9-11.5; $P=0.0003$ ).

A treat-to-target approach is therefore becoming the standard of care, but most centers are a long way off in implementing this approach systematically for all patients, in part, because of practical, resource-related factors such as the cost of fecal calprotectin or endoscopy. The gap may also be related to patients' perspective on regular endoscopies, especially if patients have not been informed that the principal treatment goal is to achieve mucosal healing.

\section{WHAT ARE THE KEY OUTCOME MEASURES NEEDED TO IMPROVE QUALITY OF CARE?}

Patients are central stakeholders in the journey to improve quality of care. They are also an important resource that, when given the appropriate language and tools, can act as their own data collection agents in monitoring the outcomes of their care. They can also offer insight and understanding into how all components of the quality process might be improved, including the local structure, process and outcome measures.

Hence, patients and patient associations, together with gastroenterologists, surgeons, specialist nurses, IBD registries and patient-reported outcome measure (PROM) methodologists, were convened by the ICHOM to develop a minimum standard set of outcomes for the management of patients aged 16 years and older with IBD. ${ }^{4}$ This international working group composed of 25 members, including representatives from Korea and China, used a modified Delphi method through a series of teleconferences to develop the final outcome measures. Systematic review of existing literature, registry data, patient focus groups and open review periods were used to reach consensus on a minimum set of standard outcome measures and risk adjustment variables. Outcome domains included survival and disease control (survival, disease activity/remission, colorectal cancer, anaemia); disutility of care (treatment-related complications); healthcare utilisation (IBD-related admissions, emergency room visits); and patient-reported outcomes (including quality of life, nutritional status and impact of fistulae) measured at baseline and at 6- or 12-month intervals. A single PROM (the IBD-control questionnaire) was recommended in the standard set and minimum risk adjustment data (demographics, basic clinical information and treatment factors) collected at baseline and annually were included. The key recommendation was the measurement of quality of life using the IBD-control questionnaire, which can readily be introduced into current practice in paper or electronic form. ${ }^{35}$

These outcome measures provide a common language through which the international IBD community can collect and report internationally comparable data on patient-centred outcomes towards the improvement of the value of care, and Asia could lead the way in this endeavor.

\section{HOW CAN THESE PRINCIPLES BE APPLIED IN ASIA TO IMPROVE THE QUALITY OF IBD CARE?}

The incidence of IBD in Asia, although still remaining lower than in Western countries, is increasing. ${ }^{36,37}$ In recent years, more and more gastroenterologists in Asia have become aware of the importance of improving the quality of IBD care, including the need for specialized care (IBD specialists and dedicated IBD nurse practitioners and educators), patient-centric ho- 
listic care, and an MDT approach. However, there is still room for greater awareness and understanding of IBD among both patients and doctors in the region. The medical resources available to specialist for IBD care may vary greatly between countries, depending on the economic resources allocated to healthcare and the national healthcare system, including those for reimbursement and healthcare delivery. Additionally, many Asian countries, especially those in East Asia, have a vertical Confucian culture that could lead to different doctor-patient relationships compared to Western countries.

Given these challenges, countries in Asia still need to establish the necessary structures and processes, and to integrate these quality measures into clinical care ${ }^{38}$ As a strength, the advanced information technology available in many Asian countries can facilitate the establishment of these required structures and processes, and should therefore be maximized. A good example is the recent use in Korea of a mobile webbased monitoring system to monitor the symptoms of CD patients and identify those with poor prognosis based on symptomatic disease. $^{39}$

Finally, government healthcare authorities should pay more attention to the increasing and substantial burden of IBD in societies, which have an impact substantially beyond the prevalence of the disease.

\section{CONCLUSIONS}

Quality of care measures and standards for IBD have already been established, and the next steps are the seamless integration of these measures and standards into daily healthcare delivery to IBD patients and the continuous upgrading of these standards and measure to reflect the latest scientific evidence in IBD. In addition to the establishment of the necessary structures and processes, patients should be encouraged to become more actively involved in the various phases of healthcare delivery and quality of care measurement, implementation and evaluation. Opportunities presented by developments in technology and clinical evidence should be maximized for quality of care to significantly impact patient outcomes.

\section{FINANCIAL SUPPORT}

The authors received no financial support for the research, authorship, and/or publication of this article.

\section{CONFLICT OF INTEREST}

BDY has received a research grant from Celltrion; consulting fees from Abbvie Korea, Celltrion, Daewoong Pharma., Ferring Korea, Janssen Korea, Kangstem Biotech, Kuhnil Pharm., Shire Korea, Takeda Korea, IQVIA, Cornerstones Health, and Robarts Clinical Trials Inc.; speaking fees from Abbvie Korea, Celltrion, Janssen Korea, Shire Korea, Takeda Korea, and IQVIA. However, all of these are not relevant to this article.

ST has received grant or research support from AbbVie, IOIBD, Lilly, UCB, Vifor, and Norman Collisson Foundation; consulting fees from AbbVie, Allergan, Amgen, Asahi, Biogen, Boehringer Ingelheim, Bristol-Myers Squibb, Celgene, Chemocentryx, Cosmo, Enterome, Ferring, Giuliani SpA, GlaxoSmithKline, Genentech, Immunocore, Immunometabolism, Janssen, Lilly, Merck, MSD, Neovacs, NovoNordisk, Novartis, NPS Pharmaceuticals, Pfizer, Proximagen, Receptos, Roche, Shire, Sigmoid Pharma, Takeda, Topivert, UCB, VHsquared, Vifor and Zeria; and speaker fees from AbbVie, Amgen, Biogen, Ferring and Takeda. However, all of these are not relevant to this article.

\section{AUTHOR CONTRIBUTION}

Conceptualization, methodology, project administration, visualization, writing and editing, approval of final manuscript: Ye $\mathrm{BD}$ and Travis S.

\section{REFERENCES}

1. Berry SK, Siegel CA, Melmed GY. Quality improvement initiatives in inflammatory bowel disease. Curr Gastroenterol Rep 2017;19:41.

2. Harbord M, Eliakim R, Bettenworth D, et al. Third european evidence-based consensus on diagnosis and management of ulcerative colitis. Part 2: Current management. J Crohns Colitis 2017;11:769-784.

3. Donabedian A. The quality of care. How can it be assessed? JAMA 1988;260:1743-1748.

4. Kim AH, Roberts C, Feagan BG, et al. Developing a standard set of patient-centred outcomes for inflammatory bowel disease-an international, cross-disciplinary consensus. J Crohns Colitis 2018;12:408-418.

5. Institute of Medicine. Crossing the quality chasm: a new health system for the 21st century. Washington DC: National Academies Press (US); 2001. 
6. Melmed GY, Siegel CA, Spiegel BM, et al. Quality indicators for inflammatory bowel disease: development of process and outcome measures. Inflamm Bowel Dis 2013;19:662-668.

7. Reddy SI, Friedman S, Telford JJ, Strate L, Ookubo R, Banks PA. Are patients with inflammatory bowel disease receiving optimal care? Am J Gastroenterol 2005;100:1357-1361.

8. Cohen RD, Yu AP, Wu EQ, Xie J, Mulani PM, Chao J. Systematic review: the costs of ulcerative colitis in Western countries. Aliment Pharmacol Ther 2010;31:693-707.

9. Porter ME. What is value in health care? N Engl J Med 2010; 363:2477-2481.

10. Lofland JH, Johnson PT, Ingham MP, Rosemas SC, White JC, Ellis L. Shared decision-making for biologic treatment of autoimmune disease: influence on adherence, persistence, satisfaction, and health care costs. Patient Prefer Adherence 2017; 11:947-958.

11. Veilleux S, Noiseux I, Lachapelle N, et al. Patients' perception of their involvement in shared treatment decision making: key factors in the treatment of inflammatory bowel disease. Patient Educ Couns 2018;101:331-339.

12. Kelso M, Feagins LA. Can smartphones help deliver smarter care for patients with inflammatory bowel disease? Inflamm Bowel Dis 2018;24:1453-1459.

13. Porter ME, Lee TH. From volume to value in health care: the work begins. JAMA 2016;316:1047-1048.

14. Hibi T, Panaccione R, Katafuchi M, et al. The 5C concept and $5 \mathrm{~S}$ principles in inflammatory bowel disease management. J Crohns Colitis 2017;11:1302-1308.

15. Morar P, Read J, Arora S, et al. Defining the optimal design of the inflammatory bowel disease multidisciplinary team: results from a multicentre qualitative expert-based study. Frontline Gastroenterol 2015;6:290-297.

16. Lee CK, Melmed GY. Multidisciplinary team-based approaches to IBD management: how might "One-Stop Shopping” work for complex IBD care? Am J Gastroenterol 2017;112:825-827.

17. Calvet X, Panés J, Alfaro N, et al. Delphi consensus statement: quality indicators for inflammatory bowel disease comprehensive care units. J Crohns Colitis 2014;8:240-251.

18. Yang SK, Hong M, Baek J, et al. A common missense variant in NUDT15 confers susceptibility to thiopurine-induced leukopenia. Nat Genet 2014;46:1017-1020.

19. Lee KM, Kim YS, Seo GS, Kim TO, Yang SK; IBD Study Group of the Korean Association for the Study of Intestinal Diseases. Use of thiopurines in inflammatory bowel disease: a consensus statement by the Korean Association for the Study of Intestinal Diseases (KASID). Intest Res 2015;13:193-207.
20. Tkacz J, Brady BL, Meyer R, Lofland JH, Ruetsch C, CoelhoPrabhu N. An assessment of the AGA and CCFA quality indicators in a sample of patients diagnosed with inflammatory bowel disease. J Manag Care Spec Pharm 2015;21:1064-1076.

21. Song HK, Lee KM, Jung SA, et al. Quality of care in inflammatory bowel disease in Asia: the results of a multinational webbased survey in the 2nd Asian Organization of Crohn's and Colitis (AOCC) meeting in Seoul. Intest Res 2016;14:240-247.

22. Bryant RV, Costello SP, Schoeman S, et al. Limited uptake of ulcerative colitis "treat-to-target" recommendations in realworld practice. J Gastroenterol Hepatol 2018;33:599-607.

23. Nakase H, Keum B, Ye BD, Park SJ, Koo HS, Eun CS. Treatment of inflammatory bowel disease in Asia: the results of a multinational web-based survey in the 2nd Asian Organization of Crohn's and Colitis (AOCC) meeting in Seoul. Intest Res 2016;14:231-239.

24. Ooi CJ, Makharia GK, Hilmi I, et al. Asia-Pacific consensus statements on Crohn's disease. Part 2: Management. J Gastroenterol Hepatol 2016;31:56-68.

25. Neary B, Doherty G, Rafter N, Gibson D. A structured care pathway improves quality of care for acute severe ulcerative colitis. J Crohns Colitis 2017;11(Suppl 1);S257-S258.

26. Walsh AJ, Weltman M, Burger D, et al. Implementing guidelines on the prevention of opportunistic infections in inflammatory bowel disease. J Crohns Colitis 2013;7:e449-e456.

27. Allen PB, Olivera P, Emery P, et al. Review article: moving towards common therapeutic goals in Crohn's disease and rheumatoid arthritis. Aliment Pharmacol Ther 2017;45:1058-1072.

28. Hanauer SB, Feagan BG, Lichtenstein GR, et al. Maintenance infliximab for Crohn's disease: the ACCENT I randomised trial. Lancet 2002;359:1541-1549.

29. Frøslie KF, Jahnsen J, Moum BA, Vatn MH; IBSEN Group. Mucosal healing in inflammatory bowel disease: results from a Norwegian population-based cohort. Gastroenterology 2007; 133:412-422.

30. Atreya R, Neurath MF. Current and future targets for mucosal healing in inflammatory bowel disease. Visc Med 2017;33:8288.

31. Peyrin-Biroulet L, Sandborn W, Sands BE, et al. Selecting Therapeutic Targets in Inflammatory Bowel Disease (STRIDE): determining therapeutic goals for treat-to-target. Am J Gastroenterol 2015;110:1324-1338.

32. Colombel JF, Panaccione R, Bossuyt P, et al. Effect of tight control management on Crohn's disease (CALM): a multicentre, randomised, controlled phase 3 trial. Lancet 2018;390:27792789. 
33. Khanna R, Bressler B, Levesque BG, et al. Early combined immunosuppression for the management of Crohn's disease (REACT): a cluster randomised controlled trial. Lancet 2015; 386:1825-1834.

34. Bouguen G, Levesque BG, Pola S, Evans E, Sandborn WJ. Endoscopic assessment and treating to target increase the likelihood of mucosal healing in patients with Crohn's disease. Clin Gastroenterol Hepatol 2014;12:978-985.

35. Bodger K, Ormerod C, Shackcloth D, Harrison M; IBD Control Collaborative. Development and validation of a rapid, generic measure of disease control from the patient's perspective: the IBD-control questionnaire. Gut 2014;63:1092-1102.
36. Thia KT, Loftus EV Jr, Sandborn WJ, Yang SK. An update on the epidemiology of inflammatory bowel disease in Asia. Am J Gastroenterol 2008;103:3167-3182.

37. M'Koma AE. Inflammatory bowel disease: an expanding global health problem. Clin Med Insights Gastroenterol 2013;6:3347.

38. Berry SK, Melmed GY. Quality indicators in inflammatory bowel disease. Intest Res 2018;16:43-47.

39. Kim ES, Kim SK, Jang BI, et al. Disease activity patterns recorded using a mobile monitoring system are associated with clinical outcomes of patients with Crohn's disease. Dig Dis Sci 2018; 63:2220-2230. 